\title{
La innovación institucional en la Unión Europea y su impacto en la naturaleza de la Unión como actor de seguridad*
}

\author{
Institutional innovation in the European Union and its impact on the \\ nature of the EU as a security actor \\ Natividad Fernández Sola** \\ Profesora Titular de Derecho Internacional y Relaciones Internacionales \\ y profesora Jean Monnet de la Universidad de Zaragoza
}

\begin{abstract}
Sumario: I. Consideraciones introductorias sobre las reformas institucionales en relación a la Política Común de Seguridad y Defensa introducidas por el Tratado de Lisboa.-II. El Servicio Europeo de Acción Exterior. Análisis del significado y consecuencias de su estructura orgánica. Algunos déficits. - III. Propuestas de cambio sugeridas por la práctica subsiguiente del Servicio Europeo de Acción Exterior.-IV. La naturaleza de la UE como actor de seguridad ¿depende de las instituciones? $-\mathrm{V}$. Conclusiones.
\end{abstract}

Resumen: Este trabajo analiza el peso institucional en la conformación de la UE como actor de seguridad. Centra su atención sobre el Servicio Europeo de Acción Exterior (SEAE) como instrumento esencial al servicio del Alto Representante de la UE para asuntos exteriores y política de seguridad. Repasa algunas deficiencias detectadas en su funcionamiento que repercuten en la Política Común de Seguridad y Defensa y, en general, en la toma de decisiones de política exterior. La reforma formal del SEAE comenzó su andadura; junto a ello, métodos informales de colaboración inter-servicios y de comunicación interinstitucional han sido adelantados con carácter previo a otros cambios legales o formales en la estructura del SEAE y a la reconsideración de la PCSD a la que debía proceder el Consejo Europeo de junio de 2015.

Palabras clave: Servicio Europeo de Acción Exterior (SEAE), procedimientos de trabajo, órganos militares de la UE, Política Común de Seguridad y Defensa, actor de seguridad

Abstract: This paper analyses the importance of the institutional framework for the EU's consolidation as a security actor. The focus is on the EEAS as the main tool for the High Representative. It highlights some of the EEAS's weaknesses revealed over time. These have an impact on the CSDP and, in general, on the foreign policy decision-making process. Formal review of the EEAS already started.

* Recibido el 20 de marzo de 2015, aceptado el 27 de mayo de 2015.

** Grupo de investigación Estudios Europeos e Internacionales, Gobierno de Aragón. 
At the same time, informal methods of inter-services cooperation have been implemented before other legal or formal changes of the EEAS structure and the CSDP review that was expected for the European Council in June 2015.

Keywords: European External Action Service (EEAS), working arrangements, EU's military bodies, Common Security and Defence Policy, security actor.

\section{Consideraciones introductorias sobre las reformas institucionales en relación a la Política Común de Seguridad y Defensa introducidas por el Tratado de Lisboa}

Si el objetivo esencial del Tratado de Lisboa, última enmienda de los Tratados constitutivos, era fomentar el estatuto de la Unión Europea (UE) como actor mundial, una vía relevante para conseguirlo era garantizar la coherencia, la consistencia y la unidad de acción exterior europea. El principal instrumento para conseguirlo fue la «recreación» del Alto Representante de la UE para asuntos exteriores y política de seguridad y el establecimiento del Servicio Europeo de Acción Exterior (SEAE) para asistirle.

No debe olvidarse, sin embargo, que los factores institucionales sólo son una parte de los condicionantes de la UE para realzar su papel internacional, tan decaído, y, en especial, como actor de seguridad mundial. Factores contextuales jugarán un papel tan destacado como los primeros; aunque será en éstos en los que centraremos nuestro análisis partiendo de la premisa de que, en las relaciones internacionales, a menudo las normas y actores internos son tan importantes como el contenido ${ }^{1}$. Se tomará también en consideración la reforma formal propuesta por la anterior Alta Representante de la Unión Europea para asuntos exteriores y política de seguridad y las modificaciones de procesos seguidos en la práctica adelantados por la Alta Representante Mogherini. Con todo ello, se intentará realizar un balance del peso de los cambios institucionales sobre la Política Común de Seguridad y Defensa $\left(\right.$ PCSD) ${ }^{2}$ y sobre la consolidación de la UE como actor de seguridad global.

${ }^{1}$ RAMOPOULOS, T. y ODERMATT, J., «EU Diplomacy: Measuring Success in Light of the Post-Lisbon Institutional Framework», en Boening, A., Kremer, J. F. y van Loon, A. (eds.), Global Power Europe. Theoretical and Institutional Approaches to the EU's External Relations, vol.1, Springer Verlag, 2013, p. 19.

${ }^{2}$ En particular tras las conclusiones del Consejo Europeo de 20 diciembre de 2013, EUCO 217/13. 
Una de las áreas en las que el Tratado de Lisboa sobre la Unión Europea, vigente desde el 1 de diciembre de 2009, enmendó los Tratados de Roma de 1957 y el Tratado de Unión Europea de 1991 fue la de la Política Exterior y Seguridad Europea.

Se culminaba de este modo un largo proceso de reforma institucional de la política exterior europea que había comenzado con la formalización en 1970 de la Cooperación Política Europea, su posterior institucionalización en el Acta Única Europea de 1987 y su transformación en pilar intergubernamental de la UE como Política Exterior y de Seguridad Común con el Tratado de Maastricht vigente desde 1993.

Coherencia, consistencia y unidad en la acción exterior europea ha sido la meta de la acción exterior de la UE desde el Tratado de Maastricht. El Tratado de Lisboa intentó dar una respuesta a ello mediante la creación de un Servicio de Acción Exterior (artículo 27.3 TUE) ${ }^{3}$, a modo de cuerpo diplomático europeo, con el objetivo de asistir a un nuevo Alto Representante configurado como miembro de la Comisión y, a la vez, encargado de la dirección y coordinación del Consejo de Asuntos Exteriores.

Este nuevo órgano en el engranaje institucional de la UE suscitó grandes expectativas ${ }^{4}$ por el realismo de su concepción que descansaba en su cooperación estrecha con los servicios diplomáticos de los Estados miembros y en una composición mixta incluyendo funcionarios, tanto del Consejo como de la Comisión y diplomáticos nacionales destacados en el Servicio. De este modo, se quería poner fin a la multitud de centros en donde

3 Además, el Tratado de Lisboa dispone de una única personalidad jurídica internacional a la UE, en vez de las anteriores correspondientes a la Comunidad Europea, al EURATOM y a la propia UE (FERNÁNDEZ SOLA, N., «La subjetividad internacional de la Unión Europea», Rev. Derecho Comunitario Europeo, vol. 6-11, 2002, pp. 85-112); asimismo, establece los principios generales que deben regir la acción exterior y sus fines, tras una desaparición aparente de los pilares de acción de la Unión, aunque mantiene las normas y procedimientos específicos de la PESC y de la PCSD. Sobre el limitado papel de la Comisión y del Parlamento Europeo y la exclusión del Tribunal de Justicia para decidir sobre prácticamente todas las decisiones adoptadas en el campo en estas políticas, de acuerdo con el artículo 24, de la PESC y de la PCSD, ver MISSIROLI, A. «The New EU Foreign Policy System after Lisbon: A Work in Progress», European Foreign Affairs Review, 15, 2010, pp. 427-452; VAN ELSUWEGE, P. y MERKET, H., «The Role of the Court of Justice in ensuring the Unity of the EU's External Representation» en Blockmans, S. y Wessel, R. A. (eds.), Principles and practices of EU external representation: Selected legal aspects, La Haya, CLEER Working Papers n. ${ }^{\circ}$ 2012/4.

${ }^{4}$ FERNÁNDEZ SOLA, N., El Servicio de Acción Exterior de la Unión Europea, Doc. Trabajo R.I. Elcano n. ${ }^{\circ}$ 46/2008, 10.11.2008. Ver también ALDECOA LUZÁRRAGA, F. (coord.), La diplomacia común europea. El Servicio Europeo de Acción Exterior, Marcial Pons ed., Madrid, 2011 y CORNAGO, N. y GUINEA, M. (coords.), Cuadernos Europeos de Deusto, número monográfico, n. ${ }^{\circ} 44,2011$. 
se formulaba y ponía en práctica la acción exterior de la Unión Europea ${ }^{5}$ fruto de una compleja y fragmentada estructura constitucional pero dejando, al mismo tiempo, vías para una participación significativa de los Estados miembros, siempre reticentes a ceder competencias en materia de política exterior y de seguridad, reducto último de su soberanía entendida en sentido clásico.

La decisión del Consejo sobre la organización y funcionamiento del SEAE, de 26 de julio de $2010^{6}$ dio paso a la operatividad del Servicio el 1 de enero de 2011 con la transferencia del personal correspondiente del Consejo y de la Comisión. Su estructura queda configurada por una administración central y las Delegaciones de la Unión Europea (UE) en terceros países y Organizaciones internacionales cuyo personal, en gran parte, pertenece al SEAE. La administración central, gestionada por el Secretario General ejecutivo está organizada en Direcciones generales dedicadas a áreas geográficas y a temas transversales o áreas multilaterales. Tanto la política de desarrollo, la de vecindad, la política de seguridad y construcción de la paz, los derechos humanos y la promoción de la democracia están reflejados en dicha estructura. Si bien parte de la antigua Dirección General de Desarrollo de la Comisión no se transfirió al SEAE y se fundió con la Dirección General de Cooperación Exterior en la nueva Dirección General de Cooperación al Desarrollo ${ }^{7}$. También han seguido en manos de la Comisión las políticas de ampliación, comercio, asistencia humanitaria y protección civil. Por su parte, del Secretariado del Consejo se transfirió al SEAE el personal de la Dirección General de Asuntos Exteriores y político-militares, por tanto, las estructuras de PCSD y gestión de crisis.

Esta división suscitó muchas cuestiones acerca de la naturaleza supranacional o intergubernamental del órgano que regía la política exterior y, en consecuencia, la deriva que adoptaría ésta ${ }^{8}$.

${ }^{5}$ Hasta 22 órganos europeos tenían una u otra competencia en la política exterior y de seguridad común, señalan Howorth y Le Gloannec; situación que les lleva a reconocer la lógica institucional tras la creación del SEAE. HOWORTH, J. y LE GLOANNEC, A.-M., «The Institutional Logic behind the EEAS», en European Policy Center, The EU Foreign Service: How to build a More Effective Foreign Policy?, EPC Working Paper n. ${ }^{\circ} 28,2007$, pp. 28-34.

${ }^{6}$ Decisión del Consejo 2010/427/EU de 26 de julio de 2010 por la que se establece la organización y funcionamiento del Servicio Europeo de Acción Exterior, DO L 201, de 3.8.2010.

${ }^{7}$ La gestión de los programa de cooperación al desarrollo sigue bajo la responsabilidad de la Comisión. El artículo 9 de la Decisión del Consejo de 26 de julio de 2010, ibidem. Respeta los respectivos roles de la Comisión y del SEAE en la programación de esta política.

8 SMITH, M. E. «The European External Action Service and the Security-Development Nexus: organizing for Effectiveness or Incoherence?», Journal of European Public Policy, vol. 20, issue 9, 2013, pages 1299-1315. 
La inclusión de los órganos de la Política Común de Seguridad y Defensa en el Servicio Europeo de Acción Exterior hace de éste el principal elemento institucional para analizar dicha política. Por otro lado, las debilidades e inconsistencias de la Política Común de Seguridad y Defensa de una Unión Europea incompleta no pueden disimularse por un «Cuerpo Diplomático europeo» al servicio de tal política.

Conscientes de la necesidad de adaptación del Servicio Europeo de Acción Exterior ${ }^{9}$ a las necesidades percibidas en más de tres años de funcionamiento, los Estados miembros previeron su revisión para el año $2013^{10}$ y, desde entonces, se siguen analizando y poniendo en práctica reorganizaciones y nuevos métodos de trabajo que facilitan la coherencia y, por tanto, el estatuto de actor internacional de seguridad de la Unión Europea.

\section{El Servicio Europeo de Acción Exterior. Análisis del significado y consecuencias de su estructura orgánica. Algunos déficits con repercusión en la Política Común de Seguridad y Defensa}

El SEAE se configura como órgano funcionalmente autónomo, con función de apoyo al Alto Representante de la $\mathrm{UE}^{11}$ quien, tras el citado Tratado, suma a las competencias de las que gozaba el cargo homónimo en versiones anteriores de los Tratados, la mayoría de las correspondientes hasta entonces al Comisario encargado de relaciones exteriores y las funciones exteriores del país que ejercía la presidencia rotatoria del Consejo (artículo 18 TUE). El Alto Representante, originalmente designado en el borrador de Tratado constitucional como Ministro Europeo de Asuntos Exteriores ${ }^{12}$, queda de este modo configurado como vínculo institucional entre el Consejo y la Comisión en asuntos de política exterior europea.

${ }^{9}$ La decisión del Consejo 2010/427/EU, de 26 de julio que establece la organización y funcionamiento del Servicio Europeo de Acción Exterior prevé en su artículo 13.3 que el Alto Representante de la UE para Asuntos Exteriores y Política de Seguridad estudie una revisión de la misma para mediados de 2013, op. cit., nota 6.

${ }^{10}$ La propia Política Común de Seguridad y Defensa es objeto de reconsideración desde el Consejo Europeo de diciembre de 2013, op. cit., nota 2.

11 A lo largo del trabajo utilizaremos el término «Alto Representante», pese a que su actual titular sea una mujer, como denominación genérica de la institución. Sólo se especificarán las referencias a alguna de sus dos titulares hasta el momento.

12 BROK, E., The External Representation of the European Union, European Convention Working Group VII, working document 26, 4781; BROK, E., VAN DER LINDEN, R., CUSHNAHAN, J., LAMASSOURE, A., European Convention Working Group VII, working document 46,5331 . 
El Servicio Europeo de Acción Exterior constituye, desde su composición a su organización, el mejor reflejo de la voluntad de coherencia en la política exterior de la UE aunando los servicios de relaciones y política exterior respectivamente antes separados de Comisión y Consejo y poniéndolos bajo la égida del nuevo Alto Representante.

No ahondaremos en el problema con el que se enfrenta la figura del Alto Representante, e indirectamente el SEAE, que es la confusión no solventada acerca de la representación internacional de la UE y la asignación de poderes en la acción exterior entre el Presidente del Consejo Europeo (artículos 15.5 y 6 TUE), el Alto Representante ${ }^{13}$ y el Presidente de la Comisión (artículo 17.6 TUE). El resultado práctico, máxime cuando la Comisión mantiene su propio personal para la preparación de las competencias exteriores que retiene, es que pueda producirse alguna descoordinación entre una acción de política exterior, de seguridad y defensa y una acción de cooperación al desarrollo, por ejemplo. La falta de delimitación competencial acerca de los distintos ámbitos materiales de la acción exterior igualmente podría llevar a la situación anterior al Tratado de Lisboa ${ }^{14}$, aunque en los temas de seguridad y defensa el riesgo es menor; la ambigüedad en este terreno sólo parece estar presente cuando se abordan operaciones con componentes civiles o para los que sean precisos recursos que gestiona la Comisión. Pese a estas prevenciones, los balances de actividad del SEAE inciden en el efecto benéfico de su existencia sobre la coherencia y continuidad de la acción exterior de la UE ${ }^{15}$.

Más de cuatro años de funcionamiento han revelado, junto a aspectos positivos, incluso por su mera existencia, algún problema motivado por un choque de personalidades entre los antiguos funcionarios europeos encargados de la política exterior, tanto en el propio Servicio como en la Comisión acerca de las respectivas competencias de política exterior y los recursos disponibles ${ }^{16}$. También por el elevado número de funcionarios de alto

13 Al Alto Representante corresponde la presidencia del Consejo de Asuntos Exteriores y la dirección de la PESC y de la PCSD, una vicepresidencia de la Comisión (artículos 18 y 27 TUE), la propuesta de nombramiento de representantes especiales (artículo 33 TUE) y la dirección del SEAE (artículo 27.3 TUE).

${ }^{14}$ FERNÁNDEZ SOLA, N., «The Stakes of the European External Action Service. What is needed in order to become a European Diplomatic Corps?», en BENEYTO PÉREZ, J. M. (coord.), La Unión Europea como actor global. Las nuevas dimensiones de la politica exterior europea, Universidad CEU-San Pablo, Madrid, siglo XXI editores, 2011, pp. 49-67; RAMOPOULOS, T. y ODERMATT, J. «EU Diplomacy: Measuring Success...», op. cit., nota 1, p. 20.

15 Ver informes anuales del Servicio Europeo de Acción Exterior. EEAS, Report by the High Representative to the European Parliament, the Council and the Commission, 22.12.2011).

${ }^{16}$ SMITH, M. E., «The European External Action Service and the Security-Development Nexus...», op. cit., nota 8. 
nivel o políticos que supuso una dura negociación entre Estados miembros acerca de dicha selección y que incidió en la estructura del Servicio. De hecho, el informe sobre la reforma del $\mathrm{SEAE}^{17}$ proponía reducir el número de posiciones de altos funcionarios e incrementar las responsabilidades de los Directores. Estas deficiencias coyunturales se han reflejado en dificultades para el establecimiento de agenda por parte del SEAE ${ }^{18}$.

En su momento señalamos la innecesaria rigidez de un funcionamiento dividido de forma tajante en áreas geográficas y el obstáculo para funciones transversales entre ellas y con las estructuras responsables de misiones y operaciones de la Política Común de Seguridad y Defensa. La propuesta de reforma del SEAE presentada por la, entonces, Alta Representante, Catherine Ashton, contemplaba la posibilidad de combinar puestos de diferentes áreas geográficas cubiertas por los mismos instrumentos políticos. ${ }^{19}$

Son notables por otra parte los desafíos de organización y funcionamiento a los que se enfrenta el SEAE. Destacaremos la ambigua posición institucional de los Representantes Especiales, la falta de definición del papel de las Delegaciones de la UE en países terceros, el aislamiento estructural de los órganos encargados de la Política Común de Seguridad y Defensa respecto del resto del engranaje y la indefinición de las relaciones entre los Representantes Especiales, las misiones PCSD y las Delegaciones de la UE en los territorios donde confluyen. La mayoría de estos factores inciden negativamente sobre el necesario enfoque integral de la política común de seguridad y defensa y de la misma política exterior, en general, de la Unión Europea.

En primer lugar, destacamos la ambigüedad en la regulación de los Representantes Especiales del Alto Representante quienes, sobre el papel, tienen una escasa conexión con las Delegaciones de la UE en el/los países implicados y con los servicios centrales. Situación que remonta a la aparición de la figura cuando la representación externa de la UE estaba en manos de

17 European External Action Service, EEAS Review, July 2013, p. 4; http://eeas.europa. eu/library/publications/2013/3/2013_eeas_review_en.pdf (última consulta 20 de mayo de 2015). Juncos y Pomorska atribuyen el elevado número de Directores Generales a cargo de diplomáticos nacionales a la politización durante el proceso de dotación de personal del Servicio; no a exigencias de racionalidad funcional. JUNCOS, A. E., POMORSKA, K., "In the face of adversity': explaining the attitudes of EEAS officials vis-à-vis the new service», Journal of European Public Policy, Vol. 20, No. 9, 2013, pp. 1332-1349.

18 VANHOONACKER, S. y POMORSKA, K., «The European External Action Service and Agenda setting in European Foreign Policy», Journal of European Public Policy, vol. 20, No. 9, 2013, pp. 1316-1331.

19 European External Action Service, EEAS Review, July 2013, p. 4. FERNÁNDEZ SOLA, N., «La reforma pendiente del Servicio Europeo de Acción Exterior y sus implicaciones en la política de seguridad de la Unión Europea», Rev. Instituto Español de Estudios Estratégicos, n..$^{\circ}$ 2, 2013, p. 7. 
la Comisión y su nombramiento por el Alto Representante tendía a cubrir el vacío en lugares donde los asuntos a gestionar eran de política exterior, no de competencia comunitaria. De acuerdo con el organigrama original del Servicio, los Representantes Especiales dependen directamente del Alto Representante, si bien en el organigrama de 2013 se establece un nexo con el Vicesecretario General político inexistente en la primera estructura organizativa. La estructura organizativa de 2014, sin modificaciones esenciales, coloca a los Representantes Especiales alineados bajo las respectivas áreas temáticas y regionales. Se mantiene su total aislamiento respecto de los órganos civiles y militares que planifican y dirigen la PCSD. Esta posición orgánica resulta sorpresiva por cuanto los Representantes Especiales proporcionan una información y experiencia sobre el terreno de enorme utilidad en el planeamiento, ejecución y evaluación de la PESC y de las misiones PCSD en particular ${ }^{20}$. Ese «aislamiento» hace la figura del Representante Especial más grata a los Estados miembros que tienden a identificarlos menos con los órganos centrales de la UE y verlos como una solución «más cercana» a sus intereses. Esta situación peculiar de los Representantes Especiales, que suele encontrar una solución funcional, puede ser fuente potencial de incoherencias en la acción exterior de la UE en áreas conflictivas y de importancia estratégica que, por esta razón, cuentan con un Representante Especial. Y es que, no siempre su tarea ha sido concertada con las misiones PCSD, con las Delegaciones de la UE o con las representaciones diplomáticas nacionales.

Puede citarse la presencia en Afganistán como un ejemplo poco exitoso de coordinación de la acción exterior de la UE. En el conflicto se suceden diversos Representantes Especiales desde diciembre de 2001, coincidentes con la Misión de Policía de la UE (EUPOL-Afganistán) desde 200721, con una Fuerza de Gendarmería Europea (EUROGENDFOR-Afganistán) operando desde 2009 y con la presencia diplomática de los Estados miembros, algunos de ellos participantes en la misión militar de la OTAN en el país (NATO-ISAF), liderada y organizada según los criterios estratégicos y operacionales, a veces erráticos, de los Estados Unidos de América. En aras de una concentración y coordinación de esfuerzos, el Representante Especial

${ }^{20}$ FOUÉRÉ, E., «The EU Special Representatives: A dying breed», CEPS Commentaries, 13 December 2013. http://www.eeas.europa.eu/statements-eeas/2015/150316_03_en.htm (última consulta 20 de mayo de 2015); FOUÉRÉ, E., «EU Delegations EU Special Representatives and Common Security and Defence Policy Missions: building a true cooperative relationship?, en L. N. González Alonso, (ed.), Between autonomy and cooperation: shaping the institutional profile of the European External Action Service, CLEER working papers n. ${ }^{\circ} 2014 / 16$, pp. $45-50$

${ }^{21}$ GROSS, E., «The EU in Afghanistan», en Gross, E. y Juncos, A. (eds.), EU Conflict Prevention and Crisis Management. Roles, Institutions and Policies, Oxon, Routledge, 2011. 
en Kabul es, al mismo tiempo, Jefe de la Delegación de la UE en Afganistán. Tradicionalmente ha jugado un papel político, incluso de coordinación de las posiciones de los Estados miembros sobre el terreno, pero su conexión con la misión de policía europea o con la fuerza de gendarmería ha sido escasa. ${ }^{22}$

Una mayor integración de los Representantes Especiales y su personal en la estructura del SEAE es idea que goza de amplia aceptación, entre otras razones, por el ahorro de recursos que supondría. ${ }^{23}$ Se ha sugerido que la Decisión de organización y funcionamiento del SEAE recoja la obligación de las Delegaciones de la UE de apoyar, comunicar y consultar a los Representantes Especiales; igualmente que se haga eco de la figura del Representante Especial que ejerce a la vez funciones de Jefe de la Delegación de la UE. La experiencia parece indicar la conveniencia de su mantenimiento, la coordinación con los demás actores de la acción exterior de la UE pero sin excesivas rigideces derivadas de su inclusión en un aparato burocrático, en cierta, medida farragoso y lento. ${ }^{24}$

Otra cuestión con cierta trascendencia es el aislamiento orgánico de las estructuras de la PCSD respecto al resto del SEAE. Desde el organigrama de 2013, la situación es mucho más compleja que la original, no sólo por el número de nuevas Direcciones, sino también por los siguientes cambios: CIVCOM y PMG pasaban a depender del Comité Político y de Seguridad. Las estructuras de gestión de crisis (EUMS, CMPD y CPCC) ) dejan de depender del Comité Militar y lo hacen directamente del Alto Representante. En el mismo bloque de estructuras de gestión de crisis se incluye

${ }^{22}$ La Fuerza de Gendarmería Europea está en primer lugar al servicio de la Unión Europea, pero también puede ser puesta al servicio de otras organizaciones internacionales, como la ONU, la OTAN o la OSCE. En el caso de Afganistán, la EGF se inserta en la misión de formación de la OTAN. Ver características de la Fuerza de Gendarmería en Afganistán en European Gendarmerie Force, http://www .eurogendfor.org/eurogendfor-missions/eurogendfor-afghanistan y sobre la misión de policía de la UE en Afganistán, en Unión Europea, http://europa.eu/legislation_summaries/foreign_and_security_policy/cfsp_and_esdp_implementation/ps0006_en.htm (última consulta 21 de mayo de 2015).

23 BLOCKMANS, S. et al., EEAS 2.0. Draft Recommendations for the 2013 EEAS Review, CEPS, Brussels, 2013, p. 13. Una referencia podría ser incluida en los artículos 5 y 6 de la Decisión de organización y funcionamiento del SEAE. El informe de reforma del EEAS presentado por la Alta Representante en julio de 2013 propone la inclusión plena de los Representantes Especiales en el SEAE transfiriendo su personal y presupuesto a éste.

${ }^{24}$ FOUÉRÉ, E., «The EU Special Representatives: A dying breed», op. cit., nota 20. El Comité Político y de Cooperación acepta, en su sesión de 16 de marzo de 2015, la propuesta de nombramiento de dos nuevos Representantes Especiales; uno para el proceso de paz de Oriente Medio y otro para Asia Central. Con ello se vuelven a cubrir dos regiones en las que el mandato de los anteriores Representantes Especiales había caducado sin renovarse. La tendencia implícita en esta designación es de mantener esta figura por la relevancia de su papel para los cometidos del Alto Representante. 
una división de política de seguridad y prevención de conflictos que aborda temas como las armas de destrucción masiva y convencionales, mantenimiento y construcción de la paz, mediación y sanciones, y, sin vinculación alguna, el Centro de Operaciones recientemente activado como Cuartel General Operacional de la UE ${ }^{25}$. Como órganos consultivos, se añade el Colegio Europeo de Seguridad y Defensa, el Centro de Satélites, la Agencia Europea de Seguridad y el Instituto de Estudios de Seguridad. Fuera de la estructura estricta de gestión de crisis se sitúan, como hasta ahora, la División de respuesta de crisis y coordinación operacional ${ }^{26}$ y el renombrado Centro de Inteligencia (IntCen) ${ }^{27}$.

El organigrama de noviembre de 2015, de un único Secretario General dependen tres vicesecretarios generales: el de asuntos económicos, el de asuntos políticos y el de PSCD y respuesta a crisis. Del segundo depende el Comité Político y de Seguridad. Del último y creado en la última reestructuración, dependen cuatro direcciones, las ya existentes CPCC, CMPD, el IntCen que pasa a denominarse Inteligencia y Centro de Situación y la de Política de Seguridad y Prevención de Conflictos. El Instituto de Estudios de Seguridad queda como órgano asesor del planeamiento estratégico. El Colegio Europeo de Seguridad y Defensa, el Centro de Satélites y la Agencia Europea de Seguridad quedan como órganos auxiliares de las estructuras de la PCSD sin una clara dependencia jerárquica.

Como esquema de funcionamiento, se aprecia en consecuencia un relativo aislamiento orgánico entre las estructuras de la PCSD de prevención y gestión de crisis y el resto del organigrama del Servicio Europeo ${ }^{28}$ que puede tener repercusiones negativas sobre la coherencia de la acción dentro de esta política. Esta situación tiene una base legal pues la Decisión sobre organización y funcionamiento del SEAE declara que la especificidad de estas estructuras de la PCSD y de gestión de crisis, la particularidad de sus funciones, del reclutamiento de sus miembros, el estatuto de su personal

${ }^{25}$ Ubicación extraña por la falta de vinculación con ningún otro órgano y porque el Centro de Operaciones no deja de ser una subdivisión de la división de operaciones del Estado Mayor de la UE (EUMS).

${ }^{26}$ Esta dirección se extiende con tres subdirecciones: la de planeamiento de respuesta de crisis, el Centro de situación y la de gestión consular de crisis.

${ }^{27}$ El Centro de Situación pasa a denominarse en 2011 Centro de Inteligencia y Análisis (IntCen).

${ }^{28}$ Artículo 4 de la Decisión del Consejo que establece la organización del SEAE, cit. Los trabajos preparatorios de la decisión de organización del SEAE muestran la existencia de propuestas como la de Eslovaquia para que los órganos de gestión de crisis quedaran fuera del Servicio. Finlandia, por el contrario, veía su integración en el Servicio como una mejora estructural esencial; ver BLOCKMANS, S. y HILLION, C. (eds.), EEAS 2.0: A Legal Commentary on Council Decision 2010/427/EU Establishing the Organisation and Functioning of the European External Action Service», CEPS, Brussels, 2013. 
y la dependencia directa del Alto Representante debía ser respetada ${ }^{29}$. Esta disposición encuadrada en las exigencias de los artículos 40 del TUE y 3 a 6 del TFUE recuerdan la naturaleza de la UE todavía parcialmente estructurada en pilares, aunque formalmente hayan desaparecido.

El reto para el Servicio, como exige la Decisión que establece su organización, está en asegurar la plena coordinación entre todas las estructuras del mismo. La falta de cualquier vínculo orgánico entre dichos órganos y el resto de Direcciones Generales, Direcciones y secciones que pueden estar implicadas en una respuesta integral ante las crisis, y que suscitaba dudas acerca del buen funcionamiento del SEAE, queda parcialmente amortiguada en la nueva organización con su dependencia del Vicesecretario general de PCSD, con igual rango que las demás direcciones geográficas o temáticas, pero sin un Director Ejecutivo. Y es que, en cierta medida, aquella posición aislada de los órganos de gestión de crisis es reflejo de la falta de integración de la Política Común de Seguridad y Defensa en el conjunto de la acción exterior de la Unión Europea.

Hacer frente a esta situación requeriría que la labor de dichos órganos permeara en la estructura de los Departamentos geográficos y temáticos del SEAE. De esta forma, se potenciaría realmente el enfoque integral (comprehensive approach) de la política exterior de la Unión puesto que cada actuación en una determinada región atendería tanto a la acción desarrollada desde las actuales Direcciones Generales geográficas ${ }^{30}$, como a las operaciones PCSD civiles o militares en dicha zona, exigiendo y facilitando una planificación conjunta, unos objetivos comunes, unos instrumentos coordinados y complementarios, una implicación directa de las Delegaciones de la $\mathrm{UE}^{31}$, de los Representantes Especiales si los hay y de las Embajadas nacionales, en definitiva, una visibilidad de la UE no parcelada por sectores, programas financieros, etc ${ }^{32}$.

29 Decisión del Consejo sobre organización y funcionamiento del SEAE, 2010, cit., artículo 4 .

30 Aunque sea este su nivel orgánico, a los departamentos geográficos y transversales se les denominan Direcciones de gestión (Managerial Directions), no Direcciones Generales.

${ }^{31}$ La revisión de los procedimientos de gestión de crisis sigue esta dirección al plantear que el Jefe de la Misión debe proporcionar conocimiento sobre el terreno para la fase de planeamiento de una acción PCSD y estar estrechamente asociado a la actividad relativa a dicha política en el país.

32 Un enfoque integral de la seguridad, incluso de las propias misiones CSDP, debe tomar en consideración otras áreas afines que caen de forma parcial o exclusiva dentro de las competencias de la Comisión, tales como la prevención de crisis, la protección civil, la estabilización post-conflicto, o la reforma del sector de seguridad, o las que residen en los demás departamentos del SEAE. Ver BLOCKMANS, S. y HILLION, C. (eds.), op. cit., nota 28 . 
Son claros los ejemplos de cuestiones actuales que requieren de esa coordinación o enfoque integral y que, por las inercias estructurales, siguen abordándose en diferentes órganos no siempre comunicados entre sí.

La cuestión de las estructuras de la PCSD, su configuración y relación con el resto del SEAE tiene que ser objeto de análisis en cualquier reforma del SEAE y el resultado debería ser consagrado por el Consejo Europeo si se ve plasmado en una nueva decisión sobre la organización del Servicio. Parte de la ecuación está en el ya conocido problema del establecimiento de una capacidad permanente de planeamiento y conducción de operaciones civiles y militares de la UE y la duda de si continuar o no con la activación del Centro de Operaciones a estos efectos ${ }^{33}$, con su limitado impacto y dificultad de expandirse física y conceptualmente. Pero con ello solamente se resuelve la cuestión de la estructura misma de los órganos de la PCSD, no su relación con el resto del SEAE; si bien es cierto que, al no depender de un Cuartel General Operacional nacional, la coordinación de uno europeo con el resto del Servicio debería resultar más sencilla.

La propuesta de reforma del SEAE no se pronunciaba al respecto, por ser ello más propio de la reconsideración de la PCSD, pero recogía la petición de la Alta Representante de que el Centro de Situación sea ubicado junto con el Centro de Respuesta de Emergencias de la Comisión para formar un único instrumento europeo. Esto permitiría un trabajo conjunto y continuado del SEAE y los órganos de la Comisión en este campo. En noviembre de 2015 esta propuesta no se ha materializado y el Centro de Situación ha quedado unido a los servicios de inteligencia. Igualmente la propuesta de reforma se pronunciaba a favor de mecanismos para que la experiencia del personal del Estado Mayor estuviera disponible para todos los departamentos políticos del Servicio y de incrementar las sinergias entre los expertos geográficos del Centro de Inteligencia con las Delegaciones y con los departamentos políticos del Servicio ${ }^{34}$. Fórmulas prácticas que paliarían algunas de las deficiencias actuales.

${ }^{33}$ Hay que recordar que el Centro de Operaciones de la UE, fue activado por vez primera el 23 de marzo de 2012 por el Consejo de Asuntos Exteriores para mejorar la coordinación y reforzar las sinergias civiles y militares entre las tres acciones de PCSD en el Cuerno de África: la operación militar EUNAVFOR ATALANTA, la misión de formación de la UE en Somalia (EUTM Somalia) y la misión civil (con componente de experiencia militar) EU capacity NESTOR (EUCAP NESTOR). http://www.eeas.europa.eu/csdp/structures-instruments-agencies/eu-operations-centre/index_en.htm (última consulta 21 de mayo de 2015). Tanto el organigrama de 2014 como el de noviembre de 2015, dejan al Activado Centro de Operaciones de la UE al margen del resto de la estructura PCSD.

${ }^{34}$ European External Action Service, EEAS Review, July 2013, pp. 5-6. Incluye la fórmula de los préstamos de personal por breves períodos del Estado Mayor (EUMS) a los departamentos políticos y viceversa. 
La separación teórico-institucional entre las misiones PCSD, las Delegaciones, los Representantes Especiales, las Embajadas de los Estados miembros y los socios de la UE constituye otro obstáculo a una aproximación integral a la gestión de crisis por parte de la Unión. El papel de representación asignado a las Delegaciones en terceros países y ante Organizaciones internacionales ${ }^{35}$ es relevante a la hora de promover e incrementar la acción exterior de la UE y su visibilidad.

Aunque cuenten con miembros que no pertenecen al SEAE, como por ejemplo los encargados de la política comercial, la efectividad de la labor representativa de la Unión queda garantizada por el Jefe de Delegación quien coordina las actividades de todos sus miembros. El Jefe de Delegación recibe instrucciones del Alto Representante y del SEAE, o de la Comisión para los poderes que le confieren los Tratados, y es responsable de su ejecución en el país anfitrión.

Por lo que aquí nos interesa, la acción de la UE en política exterior y de seguridad común e intervenciones humanitarias puede obtener mayor visibilidad y credibilidad con el apoyo del Jefe de la Delegación ${ }^{36}$ sobre el territorio del Estado en el que una misión de la UE se desarrolla. Aunque éste carece de competencias en el ámbito de las misiones y operaciones PCSD que se puedan desarrollar en su zona, y no participa de la cadena de mando, puede sin embargo proporcionar guía política local al jefe de la operación y éste tiene obligación de coordinar y consultar su actuación cuando la misma pueda tener un impacto en ámbito político. Además, las Delegaciones deben constituir un importante valor añadido para el Servicio Europeo de Acción Exterior si disponen de un buen servicio de información que será útil igualmente para la Comisión y para los Estados miembros ${ }^{37}$.

También se ha sugerido que las Delegaciones europeas asuman la competencia, entre otras, en protección civil, intervención en situaciones de cri-

${ }^{35}$ Las Delegaciones de la UE ante terceros Estados y Organizaciones internacionales, al no ser administración central del SEAE no tienen un reflejo en el organigrama del mismo, pero sí su regulación en la Decisión del Consejo 2010/427/EU, op. cit., nota 6.

36 Tras la entrada en vigor del Tratado de Lisboa, las Delegaciones de la UE asumieron las responsabilidades en PESC y, consecuentemente, amplían su agenda a todos los temas lo que ha permitido una relación global con los Estados anfitriones. También ha dado a su labor de coordinación una profundidad estratégica y la ha dotado de estabilidad.

${ }^{37}$ Lejos están las Delegaciones de la UE de las pretendidas Embajadas europeas cuya presencia ejercería una mayor influencia en terceros países y permitiría la preparación de iniciativas diplomáticas conjuntas. Ver MÉNDEZ DE VIGO, I., Towards the Establishment of a Common European Diplomacy, European Convention Working Group VII, working document 55, 3/XII/2002, p. 5. En la misma línea, la Declaración escrita de acuerdo con el artículo 116 del reglamento interno del Parlamento Europeo, de BROK, E. et al., Foreign Policy, Security and Defense Union. Documento n. ${ }^{\circ}$ PE 0010/2007, 31/I/2007. 
sis y asistencia humanitaria ${ }^{38}$, lo que choca con la escasez de medios económicos y personales de las Delegaciones para estas tareas.

Un acuerdo entre los Estados miembros para reconocer que el Jefe de la Delegación de la UE preside las reuniones de los representantes nacionales en un país tercero serviría como base para la coordinación de las actividades de las embajadas de los Estados miembros. Y esta es la situación que se da en lugares donde hay una misión de la Unión Europea desplegada o en áreas conflictivas como Nairobi, Kampala, Sana'a, Cairo, Trípoli... El Tratado de Lisboa permite organizar conjuntamente los esfuerzos para la evacuación de ciudadanos europeos en caso de desastres naturales o emergencias políticas. Sin embargo, casos recientes (Libia, Túnez, Egipto o Yemen) ponen de manifiesto que los Estados miembros coordinan sus esfuerzos pero no actúan conjuntamente, como Unión Europea.

Una sugerencia para la reforma del SEAE sería la introducción de un órgano de enlace con las Delegaciones, bidireccional y coordinador con las líneas generales de la política exterior de la UE en la región y globalmente.

En cuanto a las misiones diplomáticas de los Estados miembros, su cooperación con el SEAE está prevista en el Tratado (artículos 32 y 35 TUE y 221 TFUE). Esta vinculación relativamente débil puede calificarse de coyuntural por varias razones. La primera, por el esfuerzo de las Delegaciones de la UE en involucrar a los Jefes de Misión de las distintas Embajadas nacionales en la búsqueda de posiciones comunes y, de alguna forma, en la toma de decisiones europeas sobre el país en cuestión. La segunda, por la adaptación progresiva de los Estados miembros a la existencia de un nuevo órgano de representación del conjunto de la Unión, el SEAE, y de unas Delegaciones de la UE que, más que competencia a la tradicional diplomacia nacional, constituyen un complemento eficaz a la labor diplomática de los Estados miembros; esta fórmula ha de permitir a los Estados una paulatina trasferencia de tareas, todas las que se puedan gestionar en común ${ }^{39}$.

38 BARNIER, M., For a European Civil Protection Force: Europeaid, Mayo de 2006; informe recogido en la recomendación de la Comisión, de 5 de diciembre de 2007, sobre la reproducción del texto del artículo 20 del TCE en los pasaportes, DO L 118 de 2008. Hasta el momento, la única competencia transferida al SEAE ha sido la relativa a protección consular pero solamente a petición de los Estados miembros, tal como establece el artículo 5.10 de la decisión de 2010 que establece el Servicio, op. cit., nota 6.

39 Citaremos los ejemplos de España con la transferencia la Embajada en Yemen a los locales de la Delegación de la UE en Sana'a a finales de 2012. Lo mismo que ocurre mediante firma de un MoU entre el Ministerio de Asuntos Exteriores y de Cooperación español y el SEAE, el 18 de noviembre de 2013, para la instalación por España de una oficina de representación en la Delegación de la UE en Bakú. También Luxemburgo trasladó la suya en Etiopía a la Delegación de la UE en dicho país. Memorandum of Understanding de 10 de diciembre de 2012, Press A 568/12, Brussels, 10 December 2012. 
A pesar de ello, se hace necesaria una más nítida definición de las tareas respectivas de los servicios diplomáticos nacionales y europeo y una mayor coordinación del SEAE tanto con las misiones diplomáticas nacionales como con las Delegaciones de la UE.

Otra de las cuestiones clave para el planeamiento y aplicación de una política exterior eficaz es la disponibilidad de la mejor información sobre asuntos internacionales. El mero hecho de que un tercio del personal del Servicio proceda de los Estados miembros debería permitirle incrementar la «inteligencia política» de la Unión puesto que el SEAE debería estar en la mejor situación para suministrar la información política, como servicio común para todos los Estados miembros y las instituciones europeas.

Dentro de los órganos centrales, el Centro de la UE de análisis de inteligencia (IntCen), actúa como núcleo de la actividad del SEAE en materia de inteligencia. Genera análisis valorativos de inteligencia, principalmente estratégico-política, procedente de todas las fuentes posibles para suministrar información de alta calidad sobre seguridad pública, tanto interna como externa, al Consejo. El Centro de Inteligencia en cooperación con la división de Inteligencia del EUMS conforman una estructura funcional, la Capacidad Única de Análisis de Inteligencia (Single Intelligence Analysis Capacity - SIAC-), donde se producen análisis combinando las fuentes civiles y militares. Mucha de la inteligencia de la UE procede de las personas desplazadas sobre el terreno, sean un Representante Especial de la UE, la Delegación europea a través de un consejero político, el comandante de una operación militar o civil de la Unión (inteligencia militar operativa), pero también de los Estados miembros.

Puesto que en la mayoría de los temas el servicio de inteligencia civil y el militar trabajan conjuntamente, de acuerdo con el procedimiento establecido por el acuerdo de colaboración SIAC, no sería precisa la creación de un órgano nuevo, lo que preserva la filosofía del Estado Mayor de la UE de tener un estado mayor coherente que cubra todos los ámbitos militares evitando desgajarle la parte de inteligencia. Un hipotético servicio de inteligencia y seguridad unificado contaría con la oposición de muchos Estados por ser opuesto a las estructuras de inteligencia nacionales y por razones financieras que lo harían difícilmente realizable a corto plazo.

Otra idea a considerar sería la integración de expertos en seguridad y defensa en Delegaciones de la UE (en algunas al menos) ${ }^{40}$, consecuencia lógica de la asunción de competencias en materia de seguridad y defensa por parte

40 Por primera vez, la sugerencia de Consejeros CSDP es recogida entre las recomendaciones académicas formalmente realizadas para la revisión del SEAE en BLOCKMANS, S. et al., EEAS 2.0. Draft Recommendations..., op. cit., nota 23, p. 13. 
de la UE y la consiguiente inclusión en el SEAE de los órganos políticos y militares de dicha política. La falta de personal cualificado en las Delegaciones de la UE en esta área política esencial, obliga a que cualquier actuación en este ámbito tenga que ser organizada desde Bruselas sin la inestimable información y conocimiento de las Delegaciones sobre el terreno o con una información genérica suministrada por el Jefe de la Delegación.

La incorporación de estos expertos es deseable al menos en las Delegaciones en países donde hay operaciones de la UE y en países considerados estratégicos militarmente para la Unión. El suministro de información para el proceso de toma de decisiones, la coordinación de los Estados miembros sobre el terreno y la aplicación de las decisiones de PCSD podría estar en manos de este «consejero de seguridad y defensa» en las Delegaciones de la UE, siempre bajo la coordinación del Jefe de Misión. En este sentido se pronunció el informe sobre revisión del SEAE presentado por la Alta Representante, al proponer la extensión del programa piloto de expertos de seguridad y defensa destacados (detached security/military experts $)^{41}$.

La solución a estos déficits del SEAE revelados por la práctica desde su inicio de funcionamiento permitiría la consolidación del aparato institucional al servicio de la proyección de la UE como actor de seguridad.

\section{Propuestas de cambio sugeridas en la práctica subsiguiente del SEAE}

La reforma prevista del SEAE quedó paralizada por la anterior Alta Representante por voluntad de dejar esta tarea para su sucesora. Ésta decidió continuar adelante con la reforma que avanza lentamente y por unos cauces menos institucionalizados de lo esperado.

Algunas reformas se han materializado sin modificación de la Decisión que estableció el SEAE. Así, los procedimientos de Gestión de crisis fueron objeto de una revisión limitada en 2013. El objetivo último de la reforma, junto a la adaptación a la existencia de nuevos órganos, es facilitar el enfoque integral y la mejor integración de los aspectos civiles y militares de la gestión de crisis, en particular la mejor coordinación del SEAE y de la Comisión $^{42}$

41 European External Action Service, EEAS Review, July 2013, p. 6. En la actualidad existe una figura próxima al agregado militar en la Delegación de la UE ante las Naciones Unidas y en la Delegación de la UE ante la Unión Africana

${ }^{42}$ Los procedimientos de gestión de crisis deberán revisarse tras la Revisión del SEAE, la publicación de la Comunicación común sobre Enfoque integral y a la vista de 
No podemos desconectar las ideas sobre una reforma del SEAE de las relacionadas con la propuesta de una reconsideración de la política común de seguridad y defensa (PCSD), esperada con cierta impaciencia por los Estados miembros, y que fue objeto del Consejo Europeo de diciembre de $2013^{43}$. El Consejo Europeo de junio de 2015 da continuidad a aquél y ahondar en esta reforma necesaria por el estancamiento de la PCSD en los últimos años. Y es que, al mismo tiempo que se busca adecuar la estructura del SEAE a las necesidades de las diferentes políticas de la Unión, se persigue un impulso a la política de seguridad y defensa que la haga efectiva, creíble y un instrumento tanto para los Estados miembros como para la representación unitaria y fuerte de la UE en el mundo ${ }^{44}$.

Entre tanto, algunas mejoras se han introducido por vías más sencillas que la reforma del SEAE o incluso de los Tratados. Tal es el caso de los acuerdos de trabajo sobre la cooperación inter-servicio entre la Comisión y el SEAE ${ }^{45}$. De acuerdo con ellos, las propuestas para acciones PESC son objeto de discusión en los grupos de trabajo relevantes del Consejo (temáticos, geográficos o el Comité para los Aspectos Civiles de la Gestión de Cri-

las conclusiones de los Estados miembros para optimizar la PCSD. El enfoque integral debe presidir también las misiones civiles PCSD y las estructuras de las que dependen (propuesta de Alemania, documento oficioso «Improving Civilian CSDP Management», mayo de 2013). No es de extrañar que una de las sugerencias planteadas al Alto Representante haya sido la del desarrollo de un conjunto de herramientas de asistencia de seguridad de la UE que incluiría la reforma del sector seguridad, la formación y el equipamiento; de modo que se combinarían las estructuras, recursos e instrumentos de creación de capacidades tanto del lado militar como civil, incluso se abordaría la falta de financiación que experimenta la Unión cuando abastece de equipamiento a terceros como parte de una misión de formación (EEAS, Deputy Secretary General. Note to the Attention of the HR/VP Catherine Ashton for Decision, European Council discussion on CSDP (2013), Brussels, 22 May 2013, EEAS/DSG1/BV/am (2013) 1371555. El informe de revisión del SEAE acepta un posterior reacondicionamiento de los procedimientos de gestión de las operaciones PCSD (European External Action Service, EEAS Review, op. cit., nota 41, p. 6).

43 Ver EEAS, Deputy Secretary General, Note to the Attention of the HR/VP... op. cit., nota 42) y European External Action Service, Review 2013, op. cit., nota 41; y el Informe de la Alta Representante/Directora de la Agencia Espacial Europea sobre Política Común de Seguridad y Defensa, Preparing the December 2013 European Council on Security and Defence, de 15 de octubre de 2013.

${ }^{44}$ European Commission, Communication A New Deal for European Defence. Towards a more Competitive and Efficient Defence and Security Sector, July 2013, COM (2013) 542 final. El informe de la Comisión se centra en la industria de defensa reivindicando su competencia para su regulación y gestión. Conclusiones del Consejo Europeo de diciembre de 2013, op. cit., nota 2.

45 For specifics on the inter-service cooperation, see the Working Arrangements, SEC(2012) 48. 
sis $-\mathrm{CIVCOM}-)^{46}$. En su condición de representación de la Comisión en los grupos de trabajo, el servicio del Instrumento de Política Exterior (Foreign Policy Instrument - FPI-) es consultado por el SEAE desde las primeras etapas de una acción PESC, con exclusión de las operaciones militares, e implicado plenamente en las discusiones sobre posibles alternativas. Tras alcanzar un acuerdo político por el Comité Político y de Seguridad -COPS - de lanzar una acción PESC ${ }^{47}$, el FPI prepara la declaración de impacto presupuestario para cada una de ellas, en consulta con los servicios relevantes de la Comisión y el SEAE. El grupo de trabajo de consejeros de relaciones exteriores (RELEX) aprueba el presupuesto de la acción y, una vez que el Consejo adopta la acción PESC de acuerdo con el artículo 28 TUE, éste sirve de base para que el FPI prepare la Decisión Financiera sobre la cual consulta a la Comisión siguiendo un procedimiento acelerado. El Alto Representante, como Vicepresidente de la Comisión tiene un poder de ésta para adoptar estas decisiones financieras; poder que puede ser delegado en el Director del FPI. Este órgano pone en práctica las decisiones financieras. Las misiones PCSD civiles desplegadas sobre el terreno así como la CPCC pueden ser requeridas para facilitar asesoramiento técnico, a lo largo de todo el ciclo de programación sobre asuntos que entran en su mandato y experiencia. Si la acción PESC tiene como objetivo la imposición de sanciones, en el procedimiento decisorio participarán tanto el equipo de sanciones de la Comisión como el del SEAE ${ }^{48}$. Aunque su fusión sería deseable tras la creación del Servicio de Acción Exterior, sin embargo, legalmente resulta para algunos problemática ${ }^{49}$.

A la espera de una remodelación interna del SEAE, junto a los procedimientos de cooperación inter-servicios en la toma de decisiones PESC, se han iniciado también nuevos procedimientos para incrementar los métodos de trabajo conjuntos entre el Servicio y la Comisión. Entre otras cosas,

${ }^{46} \mathrm{El}$ informe de reforma del SEAE, EEAS Review (p. 6) pide al Consejo que reconsidere la presidencia rotativa de algunos grupos de trabajo del Consejo como los de contraterrorismo, el Athena, o el de África, Caribe y Pacífico para dejarla a la determinación del Alto Representante, en aras de una mayor coherencia política.

${ }^{47}$ Este mecanismo es exclusivo para las operaciones civiles debido a su mecanismo de financiación que maneja el Instrumento de Política Exterior (FPI). En el caso de operaciones militares, son financiadas por los Estados miembros mediante su contribución al mecanismo Athena.

48 Por el momento, solo se contempla que la Dirección de Política de Seguridad y Prevención de Conflictos implique a todos los servicios relevantes de la Comisión para tomar en consideración su experiencia técnica en el desarrollo de las medidas sancionadoras

${ }^{49}$ PORTELA, C., Sanctions and the Balance of Competences, Review of Balance of Competences, United Kingdom Government, Febrero 2013, en https://www.gov.uk/government/uploads/system/uploads/attachment_data/file/224432/evidence-clara-portela.pdf (última consulta 21 de mayo de 2015). 
se contempla dar un mayor realce a la presencia de los representantes de la Comisión en el Comité Político y de Seguridad, incluso en preparación temprana y planeamiento de la agenda o compartiendo los briefings del portavoz del PSC. Por otra parte, se harían sistemáticas las reuniones inter-servicio de los equipos de país, implicando a la Comisión, se promovería la producción conjunta de los action-trackers o documentos en los que se detallan las actividades de desarrollo de un documento estratégico, así como de comunicaciones conjuntas. Se ha acordado la realización de reuniones periódicas entre el Jefe de Gabinete de la Alta Representante en la Comisión y el Secretario General ejecutivo del SEAE, así como de éste con los Directores y Jefes de Departamento del Servicio. Este tipo de encuentros de periodicidad semanal han de garantizar el conocimiento mutuo por ambos grupos de las actividades exteriores y su acción coordinada. Una reunión semanal del Jefe de la División de Coordinación Política con el Gabinete de la Alta Representante es otro instrumento con el idéntico objetivo. Al mismo tiempo que se refuerza la presencia de la Comisión, se busca que igualmente que el SEAE esté informado de la preparación de reuniones del Grupo de Comisarios encargados de las relaciones exteriores. La preparación de las reuniones del Consejo de Ministros de Defensa se someterá a la Dirección de Gestión de Crisis y Planeamiento (CMPD). Finalmente, se busca la información precisa del Gabinete de la Alta Representante y del SEAE en los contactos e informaciones a preparar para el Presidente del Consejo Europeo y para el de la Comisión, así como para el Parlamento Europeo ${ }^{50}$.

También se comienza a reconocer la necesidad de personal en el SEAE, no sólo con perfil diplomático generalista, sino de auténticos expertos en áreas temáticas como pueden ser energía, seguridad marítima u otros. Para una política común de seguridad y defensa, el SEAE debe integrar los mejores profesionales civiles y militares. Esa necesidad de especialización es incompatible con una diplomacia europea que, tradicionalmente, ha estado ejercida por funcionarios que rotan en cometidos y áreas de actividad dispares.

Igualmente se trata de evitar la actuación free-rider de Direcciones Generales de la Comisión al negociar acuerdos o memoranda con terceros o al dar mandato a sus miembros en Delegaciones exteriores de la UE; se trataría de garantizar que el Alto Representante ejerza realmente su trabajo de coordinación asignado por el Tratado de Lisboa y, para ello, que disponga de toda la información derivada de las Delegaciones.

Finalmente, se persigue que los temas domésticos con impacto en los temas de derechos humanos y democracia en el exterior o los de coopera-

50 Algunos de ellos plasmados en un documento de trabajo del SEAE, Working Arrangements between the European External Action Service and the HR/VP's Cabinet, 2015. 
ción al desarrollo estén coordinados de forma institucional. Lo más relevante es que las decisiones más importantes se estudian en el grupo de comisarios RELEX y, por tanto, se discuten en el colegio de comisarios por lo que, siendo autónomo, el SEAE se ve más próximo a la Comisión que en el momento de su creación.

De todas estas innovaciones en los métodos de trabajo, algunas ya en funcionamiento, se desprende un mayor protagonismo de la Comisión en la actividad del SEAE disipando los temores, en su momento suscitados, sobre el excesivo intergubernamentalismo de este Servicio establecido en virtud de las previsiones del Tratado de Lisboa. Es pronto para determinar si ello ha de dar más fuerza a la UE como actor securitario global o debilitarlo por rechazo de los Estados miembros a perder protagonismo en aras de la institución más integradora.

Los cambios realizados desde mediados de 2015 en el SEAE han sido cambios estructurales que han afectado esencialmente a su cúpula, en línea con la propuesta de Ashton. Ha desaparecido el Oficial Jefe de Operaciones, número tres del Servicio, y se ha creado un tercer puesto de Vicesecretario General, encargado de seguridad y de gestión de crisis, el cual asume la antigua Dirección VII de gestión de crisis y coordinación operativa así como la responsabilidad sobre los órganos propios de la PCSD (CMPD y CPCC), y el Centro de Situación, quedando el EUMS como Dirección General coordinada con los servicios que dependen del nuevo Vicesecretario General pero independiente de éstos.

Junto al Vicesecretario General Político, el encargado de Asuntos Generales se ocupará de las relaciones con el Parlamento Europeo y el Alto Representante de las relaciones con la Comisión, de la cual es miembro ocupando la vicepresidencia de la misma.

\section{La naturaleza de la UE como actor de seguridad ¿depende de las instituciones?}

Hasta el momento y pese a las deficiencias señaladas del SEAE, propias de un organismo que inicia su andadura, pueden destacarse notables éxitos como el diálogo entre Belgrado y Pristina, el acuerdo de principio sobre el programa nuclear iraní o las operaciones realizadas en el Golfo de Adén, de lucha contra la piratería, de apoyo a Somalia y de refuerzo de las capacidades marítimas de los países de la región.

Aunque toda reestructuración del SEAE tenga un impacto sobre la capacidad de la UE como actor de seguridad global, ésta no depende tan sólo de los instrumentos institucionales sino también de los recursos asignados a esa finalidad y de las capacidades disponibles para la misma. 
En este sentido, el esfuerzo dedicado a una parte de la PESC, como es la defensa, en los países europeos conjuntamente considerados queda muy atrás del de potencias mundiales, principalmente los Estados Unidos de América y las potencias asiáticas, destacadamente China.

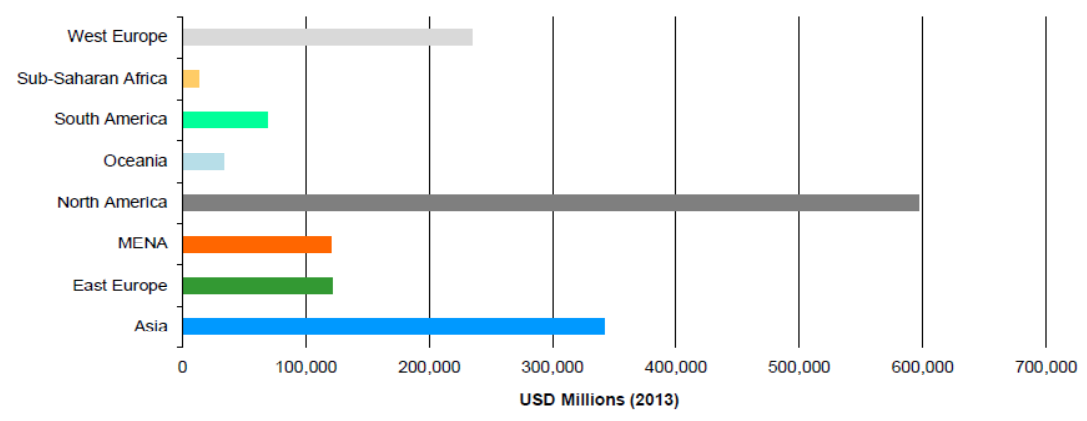

Fuente: IHS Jane's $360^{51}$.

Global Defence Expenditure (2013)

Si analizamos la situación actual con las previsiones a corto plazo, la diferencia parece agrandarse, en particular comparado el gasto europeo con el chino, el ruso y, en menor medida, el indio, en progresión ascendente.

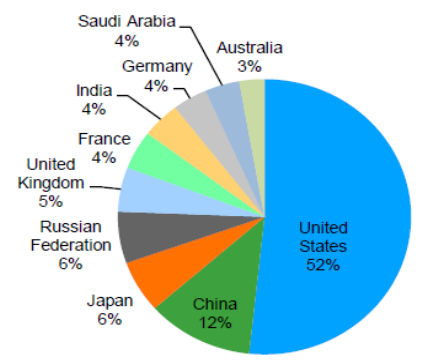

Fuente: IHS Jane's $360^{52}$.

Top Ten Defence Budgets 2013 (USD 1,117.4 billion)

${ }^{51}$ En http://www.janes.com/security/military-capabilities, (última consulta 21 de mayo de 2015).

52 Ibidem. 


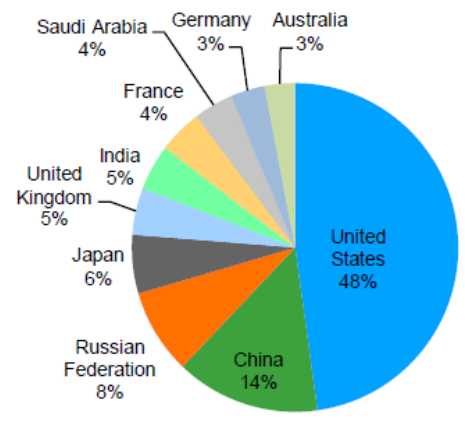

Fuente: IHS Jane's $360^{53}$.

Top Ten Defence Budgets 2017 (USD 1,173.6 billion)

La limitación de los recursos presupuestarios, además de en los presupuestos de defensa de los Estados miembros, se deja sentir muy particularmente en el de la Agencia Europea de Defensa (EDA) que sufre la consecuencia de esos recortes nacionales y de la falta de voluntad de algunos Estados de dotar de entidad competitiva a la Agencia.

Los tres elementos que permitirían reforzar la Política Común de Seguridad y Defensa, de acuerdo con el Consejo Europeo, son el aumento de la efectividad, visibilidad e impacto de esta política, la mejora del desarrollo de las capacidades de defensa y el refuerzo de la industria europea de defensa. ${ }^{54}$ Ninguna de ellas es realizable sin recursos a excepción de la visibilidad de dicha política que resulta mayor que cualquier otra proyección exterior de la UE con una menor asignación de fondos.

Globalmente considerado, el protagonismo de la UE como actor de seguridad, hoy muy limitado, depende de factores tanto institucionales como contextuales. Entre los primeros, uno es la reforma pendiente del SEAE, incluidos sus órganos militares; otros, la modificación de los procedimientos de gestión de crisis o la reforma de la PCSD. En este mismo apartado hay que señalar la necesaria adopción de una nueva Estrategia Europea de Seguridad, adaptada a las amenazas y riesgos actuales y al entorno de crisis económica de la que Europa no ha acabado de salir. Finalmente, no se puede omitir la relevancia de terminar con los recelos inter-institucionales que lastran la acción coherente, también en el campo de la seguridad y defensa.

53 Ibidem.

${ }^{54}$ Conclusiones del Consejo Europeo de diciembre de 2013, cit. 
Los factores contextuales son cuatro y de variada índole. En primer lugar el contexto de crisis financiera ha conllevado la reducción generalizada de los presupuestos de defensa de los Estados miembros hasta el punto de poder cuestionar la operatividad de muchas de las capacidades militares afectadas. En segundo lugar, la crisis política derivada de la primera y de un aparente desgaste del modelo político que ha llevado al auge de formaciones de corte más radical pone en tela de juicio la estabilidad interna necesaria para una proyección exterior fuerte; máxime cuando fuerzas con peso creciente en el Parlamento Europeo cuestionan a la propia Unión. Un factor relacionado con la crisis política de la UE es la fatiga institucional generada por sucesivas y, alguna, masivas ampliaciones del número de sus Estados miembros. Esto ha acarreado la presencia de culturas estratégicas muy diferentes y de percepciones casi antagónicas de las prioridades defensivas y securitarias europeas. Este hecho está directamente relacionado con la falta de voluntad política de una UE cada vez más heterogénea. Como resulta evidente, no es posible configurarse como actor mundial de seguridad sin una concepción mínimamente común de ésta y unas directrices de política exterior claras que guíen a la política de seguridad europea. En definitiva, en la UE conviven en la actualidad posiciones discrepantes sobre los objetivos hipotéticamente comunes de la política de seguridad y defensa y, globalmente, sobre la política exterior. Estos condicionantes actúan de forma claramente negativa para la consolidación de la UE como actor de seguridad mundial, como ha podido verificarse en el papel jugado, por ejemplo, ante la guerra en Ucrania iniciada a principios de 2014.

\section{Conclusiones}

Las deficiencias señaladas de estructura y funcionamiento del SEAE afectan negativamente al necesario enfoque integral de la política exterior y, en particular, de la PCSD de la UE. Enfoque integral que requiere coordinación entre los órganos de gestión de crisis y las demás Direcciones del Servicio, de éste con la Comisión, coordinación con los Estados miembros tanto en Bruselas como sobre el terreno y la utilización ágil de todos los instrumentos en manos de la Unión. Dicho de otra forma, la Política Común de Seguridad y Defensa es una suma de vectores, muchos de ellos, en muchas ocasiones, de sentido opuesto que representan los intereses de los Estados miembros o la orientación de diferentes instituciones de la UE. Esta dificultad viene inscrita en la naturaleza híbrida del SEAE que no es órgano intergubernamental, ni supranacional. Por esta razón, las decisiones de política de seguridad y defensa no siempre son el fruto de una aproximación integrada tomando como eje un interés estratégico común europeo. Pero lo 
que constituye una debilidad es, al mismo tiempo, una fortaleza o una oportunidad de un Servicio de carácter integrador y que puede ayudar a paliar un declive europeo como actor internacional y de seguridad que avanza rápidamente.

La importancia de llevar a buen puerto una aproximación integrada de la gestión de crisis ha de dar credibilidad, además de la visibilidad de la que ya gozan las misiones PCSD en relación con su bajo coste, si las comparamos con las actuaciones de ayuda humanitaria o de desarrollo, por ejemplo.

La revisión formal del Servicio Europeo de Acción Exterior no se ha materializado a través de una nueva decisión que reestructure su organización; más bien se han seguido mediante revisiones de su organigrama las pautas sugeridas de reducción de personal del rango más elevado, y político, y de mayor imbricación de las estructuras de seguridad y defensa con la dirección o Corporate Board del Servicio. Estos cambios, junto al reajuste de los procedimientos de trabajo internos puede incrementar la eficacia del SEAE y la coherencia de la PCSD. Las propuestas ya reseñadas y otras como la aceleración para la obtención y el incremento de recursos financieros para la preparación de acciones de política exterior, la creación de un centro de servicios logísticos y administrativos compartidos para misiones CSDP y Representantes Especiales o el reforzamiento de la capacidad de planeamiento político del SEAE ponen de manifiesto la toma de conciencia de esos problemas y de la voluntad de afrontarlos.

Hay cambios que, siendo necesarios, son de realización más compleja. Tal es el caso de la dotación a la UE de una capacidad permanente de planeamiento y conducción de operaciones civiles y militares en la estructura orgánica del SEAE, lo que indudablemente simplificaría las estructuras y procedimiento de la PCSD. Eventualmente a esta formulación podría llegarse a través de una cooperación reforzada respaldada por prácticamente la totalidad de los Estados miembros.

Otra medida necesaria sería la renovación de la Estrategia Europea de Seguridad adoptada en 2003 y, de algún modo, actualizada en 2008 pero que no responde a los desafíos de seguridad del actual momento. Las fuerzas puestas al servicio de la UE, como las nacionales, están llamadas cada vez más a planear, apoyar y ejecutar una amplia gama de funciones no tradicionales. Estas nuevas tareas militares y de seguridad, como son las misiones de estabilización o de contraterrorismo, unidas a las restricciones presupuestarias, piden una adaptación de los ejércitos que los haga extraordinariamente flexibles y capaces. Una definición de las prioridades de seguridad de la UE, en una nueva Estrategia de Seguridad Europea, permitiría esa reordenación de fuerzas para adecuarlas a las nuevas exigencias. El elevado componente polí- 
tico de esta decisión la hace particularmente delicada y necesitada de grandes dosis de liderazgo dentro de la UE como el representado en su momento por el entonces Alto Representante, Javier Solana, cuando se aprobó la, de momento, única estrategia de seguridad de la Unión.

Una lógica funcional ha llevado a una política común de seguridad y defensa en la que los intereses nacionales se incluyen en el interés europeo, sin desaparecer la política nacional de defensa para riesgos no compartidos con los socios europeos. Ahora es el momento de desarrollar de forma coherente todos los instrumentos y actores que toman parte en esta política, más allá de los exclusivamente militares. Cabe recordar que entramos en una dinámica de sustitución de la intervención militar por las misiones calladas del poder militar: conocimiento y anticipación, diplomacia militar, prevención y disuasión ${ }^{55}$. Es posible que las futuras misiones CSDP de la UE vayan en la línea de operaciones de adiestramiento o civiles y policiales, habida cuenta del éxito de las misiones tipo EUCAP y EUTM; aunque no debería desconocerse la relevancia de misiones militares con predominancia de infantería convencional ligera o de fuerzas especiales $^{56}$; es decir, la PCSD no debería descartar a priori operaciones en toda la gama de posibilidades que abre el Tratado de la UE en sus artículos 41 a 43 .

Institucionalmente, y aunque susceptible de mejoras, puede afirmarse que existe un cuerpo diplomático europeo para el diseño de la política de seguridad y unos órganos civiles y militares para la planificación y desarrollo de misiones CSDP sobre el terreno.

Sin embargo, el mejor marco institucional no puede suplir el entendimiento político necesario para superar el impasse generado por algún Estado miembro como el Reino Unido que niega cualquier posibilidad de avance en la PCSD y se opone a una mayor integración, pese a los beneficios que ello reportaría a todos en una época de peligrosos recortes en los presupuestos de defensa ${ }^{57}$; o para superar la división de enfoques estratégi-

55 Sobre el concepto de silent security, ver BLACKHAM, J. y PRINS, G., «Why Things Don't Happen: Silent Principles of National Security», RUSI Journal, vol.155, n. 4, 2010, pp. 14-22.

56 CLARK, J. L., «What Roles and Missions for Europe's Military and Security Forces in the 21st Century?», The Marshall Center Papers 7, George C. Marshall Center, 2005.

${ }^{57}$ El enfoque de la PCSD del denominado quinteto de Weimar (Francia, Alemania, España, Italia y Polonia) partidario de una mayor integración en materia defensiva y de la industria militar, o de la creación de un Cuartel General Operacional europeo para dirigir las operaciones militares de la UE, y el enfoque del Reino Unido, opuesto a iniciativas que impliquen un fortalecimiento de esta política, es reflejo de la dualidad de filosofías que se aprecian en esta política y dificultan su desarrollo. 
cos existentes entre los países europeos, en gran medida debido a sus particulares peripecias históricas.

Si el marco institucional es un requisito para la cohesión y la efectividad de la acción externa de la UE, no es el único o no es suficiente; hace falta la conciencia de la necesidad de actuar «en europeo» y la posterior determinación política de hacerlo. 